\title{
Unusual Equilibria Involving Group 4 Amides, Silyl Complexes, and Silyl Anions via Ligand Exchange Reactions
}

\author{
Xianghua Yu, ${ }^{a}$ Hu Cai, ${ }^{a}$ Ilia A. Guzei, ${ }^{b}$ Ziling Xue ${ }^{a}$ \\ ${ }^{a}$ Department of Chemistry, The University of Tennessee, Knoxville, Tennessee 37996-1600 \\ ${ }^{b}$ Department of Chemistry, The University of Wisconsin, Madison, Wisconsin 53706-1396
}

\section{Supporting Information}




\section{Experimental Section}

All manipulations were performed under a dry nitrogen atmosphere with the use of either a dry box or standard Schlenk techniques. Solvents were purified by distillation from potassium/benzophenone ketyl. Benzene- $d_{6}$, THF- $d_{8}$ and toluene- $d_{8}$ were dried over activated molecular sieves and stored under $\mathrm{N}_{2} . \mathrm{ZrCl}_{4}$ and $\mathrm{HfCl}_{4}$ (Strem) were freshly sublimed under

vacuum. The freezing point depression experiment was conducted in benzene. $\mathrm{Zr}\left(\mathrm{NMe}_{2}\right)_{4}(\mathbf{3}){ }^{7,8}$ $\mathrm{Li}(\mathrm{THF})_{2} \mathrm{SiBu}^{\mathrm{t}} \mathrm{Ph}_{2}(\mathbf{2}-\mathrm{Li}),{ }^{9}$ and $\mathrm{Li}(\mathrm{THF})_{3} \mathrm{Si}\left(\mathrm{SiMe}_{3}\right)_{3}(\mathbf{9}-\mathrm{Li})^{17}$ were prepared by the literature procedures. ${ }^{1} \mathrm{H}$ and ${ }^{13} \mathrm{C}\left\{{ }^{1} \mathrm{H}\right\}$ NMR spectra were recorded on a Bruker AC-250 or AMX-400 spectrometer and referenced to solvent (residual protons in the ${ }^{1} \mathrm{H}$ spectra). ${ }^{1} \mathrm{H}$ EXSY (Exchange Spectroscopy) spectrum was recorded at $32^{\circ} \mathrm{C}$ on a Bruker AMX-400 spectrometer. Elemental analyses were performed by Complete Analysis Laboratories Inc., Parsippany, New Jersey.

For the thermodynamic studies, the equilibrium constants $K_{\text {eq }}$ were obtained from at least two separate experiments at a given temperature, and their averages are listed. The estimated uncertainty in the temperature measurements for an NMR probe was $1 \mathrm{~K}$. The enthalpy $\left(\Delta H^{\circ}\right)$ and entropy $\left(\Delta S^{\circ}\right)$ were calculated from an unweighted nonlinear least-squares procedure contained in the SigmaPlot Scientific Graph System. The uncertainties in $\Delta H^{\circ}$ and $\Delta S^{\circ}$ were computed from the following error propagation formulas (eqs S1 and S2), ${ }^{6 \mathrm{~b}}$ which were derived from $-R T \ln K_{\text {eq }}=\Delta H^{\circ}-T \Delta S^{\circ}$.

$$
\begin{aligned}
& \left(\sigma \Delta H^{\circ}\right)^{2}=\frac{R^{2}\left(T_{\min }^{4} T_{\max }^{2}+T_{\min }^{2} T_{\max }^{4}\right)}{\left(T_{\max }-T_{\min }\right)^{4}}\left[\ln \left(\frac{K_{\mathrm{eq}(\max )}}{K_{\mathrm{eq}(\min )}}\right)\right]^{2}\left(\frac{\sigma T}{T}\right)^{2}+\frac{2 R^{2} T_{\max }^{2} T_{\min }^{2}}{\left(T_{\max }-T_{\min }\right)^{2}}\left(\frac{\sigma K_{\mathrm{eq}}}{K_{\mathrm{eq}}}\right)^{2} \\
& \left(\sigma \Delta S^{\circ}\right)^{2}=\frac{2 R^{2} T_{\min }^{2} T_{\max }^{2}}{\left(T_{\max }-T_{\min }\right)^{4}}\left[\ln \left(\frac{K_{\mathrm{eq}(\max )}}{K_{\mathrm{eq}(\min )}}\right)\right]^{2}\left(\frac{\sigma T}{T}\right)^{2}+\frac{R^{2}\left(T_{\max }^{2}+T_{\min }^{2}\right)}{\left(T_{\max }-T_{\min }\right)^{2}}\left(\frac{\sigma K_{\mathrm{eq}}}{K_{\mathrm{eq}}}\right)^{2}
\end{aligned}
$$


Preparation of $\left[\mathrm{Zr}\left(\mathrm{NMe}_{2}\right)_{5} \mathrm{Li}_{2}(\mathrm{THF})_{4}\right]^{\dagger}\left[\mathrm{Zr}\left(\mathrm{NMe}_{2}\right)_{3}\left(\mathrm{SiBu}^{\mathrm{t}} \mathrm{Ph}_{2}\right)_{2}\right]^{-}(\mathbf{1})$. Freshly sublimed $\mathrm{Zr}\left(\mathrm{NMe}_{2}\right)_{4}(\mathbf{3}, 0.300 \mathrm{~g}, 1.12 \mathrm{mmol})$ was mixed with $\mathrm{Li}(\mathrm{THF})_{2} \mathrm{SiBu}^{\mathrm{t}} \mathrm{Ph}_{2}(\mathbf{2}-\mathrm{Li}, 0.394 \mathrm{~g}, 1.08$ mmol) in a Schlenk flask. About $4 \mathrm{~mL}$ of cold toluene $\left(-10^{\circ} \mathrm{C}\right)$ was added to the mixture to dissolve the two compounds. The solution was then cooled to $30^{\circ} \mathrm{C}$ in a cold bath. Bright orange crystals gradually grew from the solution. The crystals were washed four times by cold toluene ( $2 \mathrm{~mL}$ per wash). Stripping away all volatiles afforded a yellow crystalline solid of $\mathbf{1}$ (0.407 g, $0.309 \mathrm{mmol}, 55.2 \%$ based on 3). Anal. Calcd for $\mathrm{C}_{64} \mathrm{H}_{118} \mathrm{~N}_{8} \mathrm{O}_{4} \mathrm{Si}_{2} \mathrm{Zr}_{2} \mathrm{Li}_{2}: \mathrm{C}, 58.40 ; \mathrm{H}$, 9.04. Found: C, 58.27; H, 8.91.

The equilibrium in eq 1 was observed when 1 was dissolved in THF- $d_{8} .{ }^{1} \mathrm{H}$ NMR (THF$\left.d_{8}, 400.0 \mathrm{MHz}, 25^{\circ} \mathrm{C}\right) \delta 7.49,6.97,6.87(\mathrm{~m}, \mathrm{Ph}), 3.62(\mathrm{~m}, \mathrm{THF}), 2.90\left[\mathrm{~s}, \mathrm{Zr}(\mathrm{NMe})_{4}, 3\right], 2.82[\mathrm{~s}$, $\left.\left.\mathrm{Zr}(\mathrm{NMe})_{5}\right)_{5}^{-} \mathbf{1 b}\right], 2.75\left[\mathrm{~s}, \mathrm{Zr}(\mathrm{NMe})_{3}\left(\mathrm{SiBu}^{\mathrm{t}} \mathrm{Ph}_{2}\right)_{2}{ }^{-}, \mathbf{1 a}\right], 1.77$ (m, THF), 0.950 (s, $\left.\mathrm{LiSi} u^{t} \mathrm{Ph}_{2}, \mathbf{2}\right)$, $0.862\left[\mathrm{~s}, \mathrm{Zr}\left(\mathrm{NMe}_{2}\right)_{3}\left(\mathrm{Si} B u^{t} \mathrm{Ph}_{2}\right)_{2}{ }^{-}, \mathbf{1 a}\right] ;{ }^{13} \mathrm{C}\left\{{ }^{1} \mathrm{H}\right\} \mathrm{NMR}\left(\mathrm{THF}-d_{8}, 100.6 \mathrm{MHz}, 25{ }^{\circ} \mathrm{C}\right) \delta 137.59$, 126.46, $124.41(\mathrm{Ph}), 68.23(\mathrm{THF}), 44.75\left[\mathrm{Zr}(\mathrm{NMe})_{5}{ }_{5}^{-}, \mathbf{1 b}\right], 43.43\left[\mathrm{Zr}(\mathrm{NMe})_{3}\left(\mathrm{SiBu}^{\mathrm{t}} \mathrm{Ph}_{2}\right)_{2}{ }^{-}, \mathbf{1 a}\right]$, $43.14\left[\mathrm{Zr}(\mathrm{NMe})_{4}, 3\right], 31.87\left(\mathrm{LiSiBu}^{t} \mathrm{Ph}_{2}, \mathbf{2}\right), 31.48\left[\mathrm{Zr}\left(\mathrm{NMe}_{2}\right)_{3}\left(\mathrm{SiCMe}_{3} \mathrm{Ph}_{2}\right)_{2}{ }^{-}, \mathbf{1 a}\right], 27.87$ $\left[\mathrm{Zr}\left(\mathrm{NMe}_{2}\right)_{3}\left(\mathrm{SiCMe}_{3} \mathrm{Ph}_{2}\right)_{2}{ }^{-}, \mathbf{1 a}\right], 26.36(\mathrm{THF})$.

Preparation of $\mathbf{Z r}\left(\mathrm{NMe}_{2}\right)_{3}\left[\mathbf{N}\left(\mathrm{SiMe}_{3}\right)_{2}\right]$ (6). $\mathrm{LiNMe}_{2}(3.29 \mathrm{~g}, 64.5 \mathrm{mmol})$ in THF (40 mL) at $30{ }^{\circ} \mathrm{C}$ was added dropwise to a slurry of $\mathrm{ZrCl}_{4}(5.00 \mathrm{~g}, 21.5 \mathrm{mmol})$ in $\mathrm{THF}(30 \mathrm{~mL})$ at $-30{ }^{\circ} \mathrm{C}$. The mixture was warmed with stirring to room temperature overnight. The mixture was then cooled to $-30{ }^{\circ} \mathrm{C}$, and $\mathrm{LiN}\left(\mathrm{SiMe}_{3}\right)_{2}(3.59 \mathrm{~g}, 21.5 \mathrm{mmol})$ in $\mathrm{THF}(30 \mathrm{~mL})$ at $-30{ }^{\circ} \mathrm{C}$ was added slowly. After stirring overnight, all volatiles were removed in vacuo. Hexanes $(2 \times 40 \mathrm{~mL})$ were added, and the solution was filtered. A solid was obtained after the volatiles in the filtrate were removed in vacuo. Sublimation in vacuo at $60^{\circ} \mathrm{C}$ gave a white waxy solid of 6 (6.20 g, 16.2 
mmol, yield: $75.1 \%$ ). ${ }^{1} \mathrm{H}$ NMR (benzene- $\left.d_{6}, 400.0 \mathrm{MHz}, 23^{\circ} \mathrm{C}\right) \delta 2.90,\left(\mathrm{~s}, 18 \mathrm{H}, \mathrm{N} M e_{2}\right), 0.275$ $\left[\mathrm{s}, 18 \mathrm{H}, \mathrm{N}\left(\mathrm{Si} M e_{3}\right)_{2}\right] ;{ }^{13} \mathrm{C}\left\{{ }^{1} \mathrm{H}\right\}$ NMR (benzene- $\left.d_{6}, 100.6 \mathrm{MHz}, 23{ }^{\circ} \mathrm{C}\right) \delta 42.14\left(\mathrm{~N} M e_{2}\right), 4.38$ $\left[\mathrm{N}(\mathrm{SiMe})_{2}\right) .{ }^{1} \mathrm{H}$ NMR $\left.\left(\mathrm{THF}-d_{8}, 400.0 \mathrm{MHz}, 23^{\circ} \mathrm{C}\right) \delta 2.94,(\mathrm{~s}, 18 \mathrm{H}, \mathrm{NMe})_{2}\right), 0.170[\mathrm{~s}, 18 \mathrm{H}$, $\left.\left.\mathrm{N}(\mathrm{SiMe})_{2}\right)_{2}\right]{ }^{13} \mathrm{C}\left\{{ }^{1} \mathrm{H}\right\}\left(\mathrm{THF}-d_{8}, 100.6 \mathrm{MHz}, 23{ }^{\circ} \mathrm{C}\right) \delta 42.59(\mathrm{NMe} 2), 4.55\left[\mathrm{~N}\left(\mathrm{Si}_{2} e_{3}\right)_{2}\right]$. Anal. Calcd for $\mathrm{C}_{12} \mathrm{H}_{36} \mathrm{~N}_{4} \mathrm{Si}_{2} \mathrm{Zr}$ : C, 37.55; H, 9.45. Found: C, 37.31; H, 9.28.

Preparation of $\mathbf{H f}\left(\mathrm{NMe}_{2}\right)_{3}\left[\mathbf{N}\left(\mathrm{SiMe}_{3}\right)_{2}\right]$ (8). $\mathrm{LiNMe}_{2}(2.39 \mathrm{~g}, 46.8 \mathrm{mmol})$ in THF (30 mL) at $40{ }^{\circ} \mathrm{C}$ was added dropwise to a slurry of $\mathrm{HfCl}_{4}(5.00 \mathrm{~g}, 15.6 \mathrm{mmol})$ in THF $(30 \mathrm{~mL})$ at $-40{ }^{\circ} \mathrm{C}$. The mixture was warmed with stirring to room temperature overnight. The mixture was then cooled to $-30{ }^{\circ} \mathrm{C}$, and $\mathrm{LiN}\left(\mathrm{SiMe}_{3}\right)_{2}(2.61 \mathrm{~g}, 15.6 \mathrm{mmol})$ in $\mathrm{THF}(30 \mathrm{~mL})$ at $-30{ }^{\circ} \mathrm{C}$ was added slowly. After stirring overnight, all volatiles were removed in vacuo. Hexanes $(2 \times 30 \mathrm{~mL})$ were added, and the solution was filtered. A solid was obtained after the volatiles in the filtrate were removed in vacuo. Sublimation in vacuo at $55^{\circ} \mathrm{C}$ gave a white waxy solid of $8(5.75 \mathrm{~g}, 12.2$ mmol, yield: 78.2\%). ${ }^{1} \mathrm{H}$ NMR (benzene- $\left.d_{6}, 400.0 \mathrm{MHz}, 23{ }^{\circ} \mathrm{C}\right) \delta 2.93,\left(\mathrm{~s}, 18 \mathrm{H}, \mathrm{NMe} e_{2}\right), 0.288$ $\left[\mathrm{s}, 18 \mathrm{H}, \mathrm{N}\left(\mathrm{Si} M e_{3}\right)_{2}\right] ;{ }^{13} \mathrm{C}\left\{{ }^{1} \mathrm{H}\right\}$ NMR (benzene- $\left.d_{6}, 100.6 \mathrm{MHz}, 23{ }^{\circ} \mathrm{C}\right) \delta 41.69\left(\mathrm{~N} M e_{2}\right), 4.43$ $\left[\mathrm{N}\left(\mathrm{Si} M e_{3}\right)_{2}\right] .{ }^{1} \mathrm{H} \mathrm{NMR}\left(\mathrm{THF}-d_{8}, 400.0 \mathrm{MHz}, 23^{\circ} \mathrm{C}\right) \delta 2.93,(\mathrm{~s}, 18 \mathrm{H}, \mathrm{NMe} 2), 0.157[\mathrm{~s}, 18 \mathrm{H}$, $\left.\mathrm{N}\left(\mathrm{SiMe}_{3}\right)_{2}\right] ;{ }^{13} \mathrm{C}\left\{{ }^{1} \mathrm{H}\right\}\left(\mathrm{THF}-d_{8}, 100.6 \mathrm{MHz}, 23{ }^{\circ} \mathrm{C}\right) \delta 42.05\left(\mathrm{~N} M e_{2}\right), 4.53$ [N(SiMe $\left.)_{2}\right]$. Anal. Calcd for $\mathrm{C}_{12} \mathrm{H}_{36} \mathrm{~N}_{4} \mathrm{Si}_{2} \mathrm{Hf}: \mathrm{C}, 30.59 ; \mathrm{H}, 7.70$. Found: C, 30.31; H, 7.59.

Thermodynamic studies of the equilibrium in eq 1 . In the first experiment, $\mathrm{Zr}\left(\mathrm{NMe}_{2}\right)_{4}(3$, $22.2 \mathrm{mg}, 0.0830 \mathrm{mmol}), \mathrm{Li}(\mathrm{THF})_{2} \mathrm{SiBu}^{\mathrm{t}} \mathrm{Ph}$ (2-Li, $\left.26.8 \mathrm{mg}, 0.0842 \mathrm{mmol}\right)$ and 4, 4'-dimethyl biphenyl (internal standard, $11.4 \mathrm{mg}, 0.0625 \mathrm{mmol}$ ) were added $0.428 \mathrm{~mL}$ of THF- $d_{8}$ in a Young NMR tube. In the second experiment, $\mathrm{Zr}\left(\mathrm{NMe}_{2}\right)_{4}(3,34.5 \mathrm{mg}, 0.129 \mathrm{mmol}), \mathrm{Li}(\mathrm{THF})_{2} \mathrm{SiBu}^{\mathrm{t}} \mathrm{Ph}_{2}$ (2-Li, $56.7 \mathrm{mg}, 0.178 \mathrm{mmol}$ ) and 4, 4'-dimethyl biphenyl (15.1 $\mathrm{mg}, 0.0828 \mathrm{mmol}$ ) were added 
$0.528 \mathrm{~mL}$ of THF- $d_{8}$ in a Young tube. Another solution was prepared by dissolving $\mathrm{Zr}(\mathrm{NMe})_{5} \mathrm{Li}_{2}(\mathrm{THF})_{4} \cdot \mathrm{Zr}\left(\mathrm{NMe}_{2}\right)_{3}\left(\mathrm{SiBu}^{\mathrm{t}} \mathrm{Ph}_{2}\right)_{2}(\mathbf{1}, 20.5 \mathrm{mg}, 0.0156 \mathrm{mmol})$ and 4, 4'dimethyl biphenyl $(15.1 \mathrm{mg}, 0.0828 \mathrm{mmol})$ in $0.472 \mathrm{~mL}$ of THF- $d_{8}$. The variable temperature ${ }^{1} \mathrm{H}$ NMR studies for these three solutions were carried out between $308 \mathrm{~K}$ and $243 \mathrm{~K}$.

In toluene- $d_{8}$, an equilibrium involving $\mathrm{Zr}\left(\mathrm{NMe}_{2}\right)_{4}(\mathbf{3}), \mathrm{SiBu}^{\mathrm{t}} \mathrm{Ph}_{2}{ }^{-}$(2), $\mathbf{1 a}, \mathbf{1 b}$, and

$\mathrm{Zr}\left(\mathrm{NMe}_{2}\right)_{6}{ }^{2-}(\mathbf{4})$ was observed. The ${ }^{1} \mathrm{H}$ NMR peaks of the amide ligands $\left(-\mathrm{NMe}_{2}\right)$ in $\mathbf{1 a}, \mathbf{1 b}, \mathbf{3}$, and 4 were severely overlapped, preventing accurate quantitative studies of the equilibrium. Thermodynamic studies of the equilibrium in eq 3. In the first experiment, $\mathrm{Zr}\left(\mathrm{NMe}_{2}\right)_{4}\left[\mathrm{~N}\left(\mathrm{SiMe}_{3}\right)_{2}\right](\mathbf{6}, 39.4 \mathrm{mg}, 0.103 \mathrm{mmol}), \mathrm{Li}(\mathrm{THF})_{2} \mathrm{SiBu}^{\mathrm{t}} \mathrm{Ph}_{2}(\mathbf{2}-\mathrm{Li}, 74.9 \mathrm{mg}, 0235$ $\mathrm{mmol})$ and the internal standard 4, 4'-dimethyl biphenyl (23.5 $\mathrm{mg}, 0.129 \mathrm{mmol})$ were added $0.719 \mathrm{~mL}$ of THF- $d_{8}$ in a Young NMR tube. In the second experiment, $\mathrm{Zr}\left(\mathrm{NMe}_{2}\right)_{4}\left[\mathrm{~N}\left(\mathrm{SiMe}_{3}\right)_{2}\right]$ (6, $21.1 \mathrm{mg}, 0.549 \mathrm{mmol}), \mathrm{Li}(\mathrm{THF})_{2} \mathrm{SiBu}^{\mathrm{t}} \mathrm{Ph}_{2}$ (2-Li, $\left.41.8 \mathrm{mg}, 0.131 \mathrm{mmol}\right)$ and 4, 4'-dimethyl biphenyl $(14.8 \mathrm{mg}, 0.0812 \mathrm{mmol})$ were added $0.524 \mathrm{~mL}$ of THF- $d_{8}$ in a Young NMR tube. The variable-temperature NMR studies were conducted between $303 \mathrm{~K}$ and $223 \mathrm{~K}$.

\section{Freezing point depression studies of a solution of} $\left.\left[\mathbf{Z r}\left(\mathrm{NMe}_{2}\right)_{5} \mathbf{L i}_{2}(\mathrm{THF})_{4}\right]^{\dagger} \mathbf{Z r}\left(\mathbf{N M e}_{2}\right)_{3}\left(\mathbf{S i B u}^{t} \mathbf{P h}_{2}\right)_{2}\right]^{-}(\mathbf{1})$ in benzene. The complex $\mathbf{1}(0.363 \mathrm{~g}$, $0.276 \mathrm{mmol}$ ) was dissolved in $4.043 \mathrm{~g}$ of benzene. The melting point of the solution was measured with a digit thermometer. The measurement was repeated two more times. The melting point decreased from $5.49^{\circ} \mathrm{C}$ to $4.43{ }^{\circ} \mathrm{C}$. From the freezing point depression equation $\left(K_{\mathrm{f}}=5.12{ }^{\circ} \mathrm{C} / \mathrm{m}\right): \Delta T_{\mathrm{f}}=-i K_{\mathrm{f}} m, i=3.03$.

NMR assignments for $\operatorname{Zr}\left(\mathrm{NMe}_{2}\right)_{5}^{-}(\mathbf{1 b})$ in THF- $\boldsymbol{d}_{\mathbf{8}}$. In a solution of crystals of $\mathbf{1}$ in THF- $d_{8}$ giving the equilibrium in eq 1 , assignments of ${ }^{1} \mathrm{H}$ and ${ }^{13} \mathrm{C}$ NMR spectra of $\mathrm{Zr}\left(\mathrm{NMe}_{2}\right)_{5}^{-}(\mathbf{1} \mathbf{b})$ in eq 1 were made, as discussed earlier. These assignments were supported by the following 
additional studies.

In the ${ }^{13} \mathrm{C}$ and ${ }^{1} \mathrm{H}$ spectra of a mixture (A) of $\mathrm{Zr}\left(\mathrm{NMe}_{2}\right)_{4}(\mathbf{3}, 31 \mathrm{mg}, 0.12 \mathrm{mmol})$ and $\mathrm{LiNMe}_{2}(2.9 \mathrm{mg}, 0.057 \mathrm{mmol})$ in THF- $d_{8}$, two peaks of ca. equally intensity $\left({ }^{1} \mathrm{H}: 2.89 \mathrm{ppm},{ }^{13} \mathrm{C}\right.$ : $44.74 \mathrm{ppm}$ and ${ }^{1} \mathrm{H}: 2.81 \mathrm{ppm}, 43.11 \mathrm{ppm}$, respectively) were observed. The peaks at $2.89 \mathrm{ppm}$ in ${ }^{1} \mathrm{H}$ and $44.74 \mathrm{ppm}$ in ${ }^{13} \mathrm{C}$ NMR were assigned to those of $\mathbf{3}$. When $3.2 \mathrm{mg}$ of $\mathrm{LiNMe}_{2}(0.063$ mmol; Total of the two portions: $0.120 \mathrm{mmol}$ ) was added to the mixture $(\mathbf{B})$, only the peaks at $2.81 \mathrm{ppm}$ and $43.11 \mathrm{ppm}$ in ${ }^{1} \mathrm{H}$ and ${ }^{13} \mathrm{C}$ NMR, respectively, were observed. These were assigned to be those of $\mathrm{Zr}\left(\mathrm{NMe}_{2}\right)_{5}^{-}(\mathbf{1 b})$.

When another portion of $\mathrm{LiNMe}_{2}(3.7 \mathrm{mg}, 0.073 \mathrm{mmol})$ was added to this mixture $(\mathbf{C})$, a new peak $\left({ }^{1} \mathrm{H}: 2.74 \mathrm{ppm},{ }^{13} \mathrm{C}: 46.39 \mathrm{ppm}\right)$ identified to be that of $\mathrm{Zr}\left(\mathrm{NMe}_{2}\right)_{6}{ }^{2-}(4)$ was observed along with that of $\mathrm{Zr}\left(\mathrm{NMe}_{2}\right)_{5}^{-}$(1) (1b), and that $\mathbf{B}$ (molar ratio of $\mathbf{3} / \mathrm{LiNMe}_{2}=1$ ) is predominantly $\mathbf{1 b}$. With the addition of a total of 1.6 equiv of $\mathrm{LiNMe}_{2}$ to $\mathrm{Zr}\left(\mathrm{NMe}_{2}\right)_{4}(\mathbf{3})$ in $\mathbf{C}$, it is a mixture of $\mathrm{Zr}\left(\mathrm{NMe}_{2}\right)_{5}{ }^{-}(\mathbf{1 b})$ and $\mathrm{Zr}\left(\mathrm{NMe}_{2}\right)_{6}{ }^{2-}$ (4).

Formation of $\operatorname{Zr}\left(\mathrm{NMe}_{2}\right)_{4}(\mathrm{THF})_{2}$ in toluene- $\boldsymbol{d}_{8}$. Bradley, Chisholm and coworkers have reported that, in benzene and toluene, $\mathrm{Zr}\left(\mathrm{NMe}_{2}\right)_{4}(3)$ shows a degree of oligomerization. ${ }^{7,8 \mathrm{a}, \mathrm{S} 1}$ For a mixture of $3(48.2 \mathrm{mg}, 0.180 \mathrm{mmol})$ and THF $(8.9 \mathrm{mg}, 0.12 \mathrm{mmol})$ in toluene- $d_{8}$ at $223 \mathrm{~K}$, the resonances of $\mathrm{Zr}\left(\mathrm{NMe}_{2}\right)_{4}(\mathrm{THF})_{2}$ was observed at 3.29 (THF), $2.98\left(-\mathrm{NMe} e_{2}\right.$ and 1.11 (THF) ppm in ${ }^{1} \mathrm{H}$ NMR spectrum, and 68.86 (THF), 43.36 (-NMe $)$, and 25.64 (THF) ppm in ${ }^{13} \mathrm{C}$ NMR spectrum, respectively. In addition, the NMR resonances of the dimer of $3^{8 a}\left[{ }^{1} \mathrm{H}\right.$ NMR $\delta 2.88$ (terminal $\mathrm{N} M e_{2}$ ), 2.34 (bridging $\mathrm{N} M e_{2}$ ); ${ }^{13} \mathrm{C} \delta 43.76$ (terminal and bridging $\mathrm{N} M e_{2}$ )] were observed as well. At $263 \mathrm{~K}$, the amide peaks in the ${ }^{1} \mathrm{H}$ NMR spectrum of the mixture of $\mathrm{Zr}\left(\mathrm{NMe}_{2}\right)_{4}(\mathrm{THF})_{2}$ and the dimer of $\mathbf{3}$ started to coalescence. At $296 \mathrm{~K}$, only one amide 
resonance was observed in the ${ }^{1} \mathrm{H}$ NMR spectrum.

X-ray crystal structure determination for 1 . The data for the crystal structure of 1 were collected on a Bruker AXS Smart 1000 X-ray diffractometer equipped with a CCD area detector and a graphite-monochromated Mo source $\left(K_{\alpha}\right.$ radiation, $\left.0.71073 \AA\right)$ and fitted with an upgraded Nicolet LT-2 low temperature device. A suitable crystal was coated with paratone oil (Exxon) and mounted on a hairloop under a stream of nitrogen at 173(2) K. The structure of 1 was first solved by direct methods in the triclinic space group $P-1$, and then converted to its correct monoclinic space group $C 2 / \mathrm{c}$. Non-hydrogen atoms were anistropically refined. All hydrogen atoms were treated as idealized contributions. Empirical absorption correction was performed with SADABS. ${ }^{\text {S2a }}$ In addition the global refinements for the unit cells and data reductions of the two structures were performed using the Saint program (Version 6.02). All calculations were performed using SHELXTL (Version 5.1) proprietary software package. ${ }^{\text {S2b }}$

\section{Additional References}

S1. Bradley, D. C.; Gitlitz, M. H. J. Chem. Soc. A 1969, 980.

S2. (a) Sheldrick, G. M. SADABS, A Program for Empirical Absorption Correction of Area Detector Data; University of Göttingen, Göttingen, Germany, 2000. (b) Sheldrick, G. M. SHELXL-97, A Program for the Refinement of Crystal Structures, University of Göttingen, Göttingen, Germany, 1997. 
Table S1. The equilibrium constants for eqs 1 and 3 at different temperatures. ${ }^{a}$

\begin{tabular}{ccc}
\hline & Eq. $\mathbf{~}$ & Eq. $\mathbf{3}$ \\
\hline $\boldsymbol{T}(\mathbf{K})$ & $\boldsymbol{K}_{\text {eq }} \pm \sigma \boldsymbol{K}_{\text {eq(ran) }}$ & $\boldsymbol{K}_{\text {eq }} \pm \sigma \boldsymbol{K}_{\text {eq(ran })}$ \\
\hline $308 \pm 1$ & $6.758 \pm 0.081$ & \\
$303 \pm 1$ & $7.927 \pm 0.061$ & $3.767 \pm 0.047$ \\
$298 \pm 1$ & $10.37 \pm 0.04$ & $3.999 \pm 0.037$ \\
$293 \pm 1$ & $12.88 \pm 0.03$ & $4.138 \pm 0.048$ \\
$288 \pm 1$ & $16.74 \pm 0.05$ & $4.392 \pm 0.015$ \\
$283 \pm 1$ & $21.97 \pm 0.07$ & $4.684 \pm 0.014$ \\
$278 \pm 1$ & $27.94 \pm 0.06$ & $4.974 \pm 0.060$ \\
$273 \pm 1$ & $40.77 \pm 0.06$ & $5.290 \pm 0.031$ \\
$268 \pm 1$ & $53.90 \pm 0.05$ & $5.505 \pm 0.046$ \\
$263 \pm 1$ & $72.38 \pm 0.09$ & $5.647 \pm 0.008$ \\
$258 \pm 1$ & $95.66 \pm 0.08$ & $6.129 \pm 0.033$ \\
$253 \pm 1$ & $124.83 \pm 0.08$ & $6.447 \pm 0.024$ \\
$248 \pm 1$ & $165.84 \pm 0.04$ & $6.903 \pm 0.019$ \\
$243 \pm 1$ & $242.09 \pm 0.08$ & $7.273 \pm 0.008$ \\
$238 \pm 1$ & & $7.994 \pm 0.024$ \\
$233 \pm 1$ & & $8.506 \pm 0.004$ \\
$228 \pm 1$ & & $9.278 \pm 0.014$ \\
$223 \pm 1$ & & $10.006 \pm 0.041$ \\
\hline
\end{tabular}

${ }^{a}$ The largest random uncertainty is $\sigma K_{\text {eq(ran) }} / K_{\text {eq }}=0.081 / 6.758=0.047 / 3.767=1.2 \%$ for both eqs 1 and 3. The total uncertainty $\sigma K_{\mathrm{eq}} / K_{\mathrm{eq}}$ of $5.1 \%$ was calculated from $\sigma K_{\mathrm{eq}(\mathrm{ran})} / K_{\mathrm{eq}}=1.2 \%$ and the estimated systematic uncertainty $\sigma K_{\text {eq(sys) }} / K_{\text {eq }}=5 \%$ by $\sigma K_{\text {eq }} / K_{\text {eq }}=\left[\left(\sigma K_{\text {eq(ran) }} / K_{\text {eq }}\right)^{2}+\right.$ $\left.\left(\sigma K_{\mathrm{eq}(\mathrm{sys})} / K_{\mathrm{eq}}\right)^{2}\right]^{1 / 2}$. 


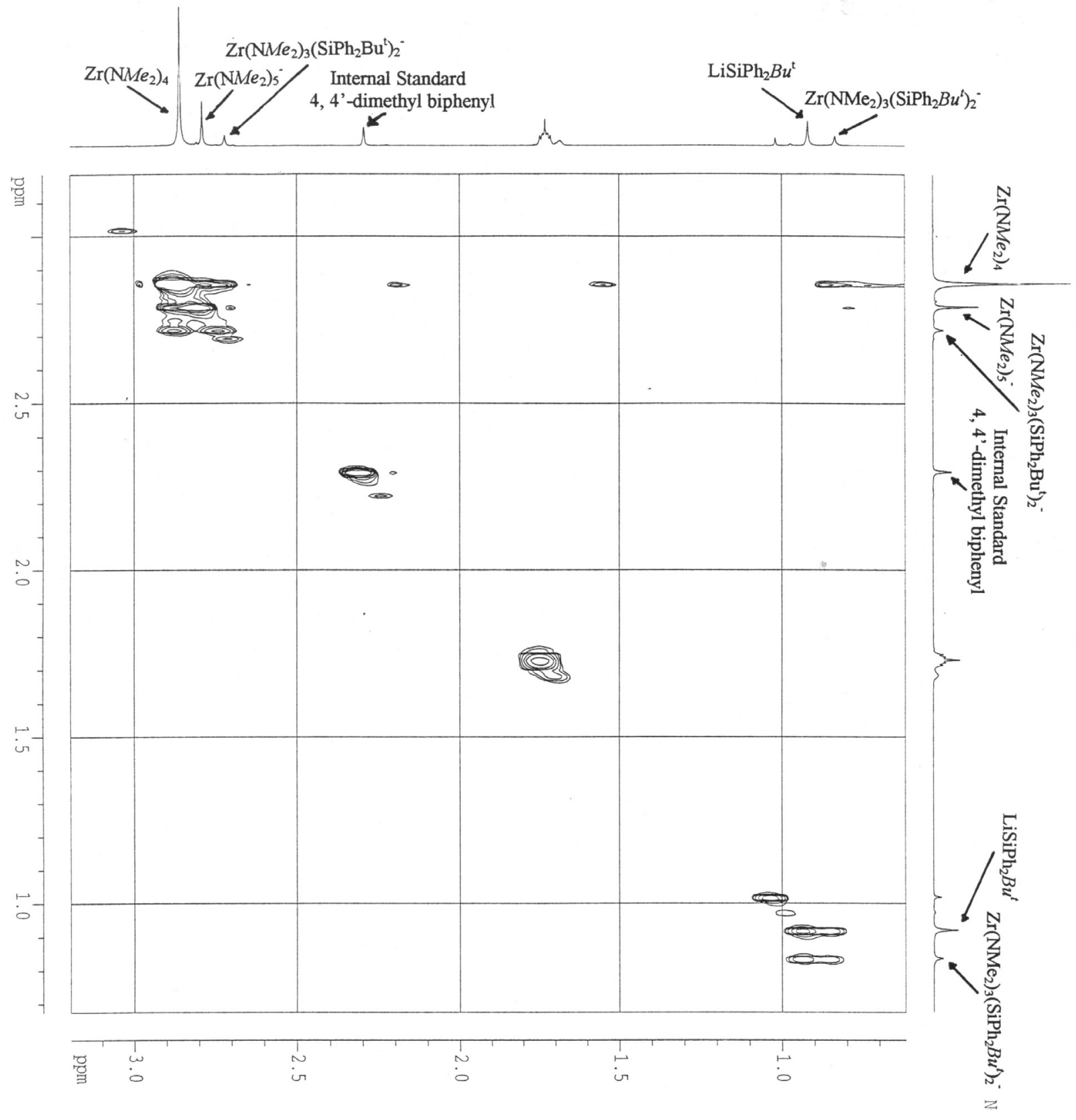

Figure S2. EXSY spectrum (THF- $\left.d_{8}, 400.0 \mathrm{MHz}, 32^{\circ} \mathrm{C}, t_{\mathrm{mix}}=2 \mathrm{~s}\right)$ of a mixture of 2 and 3. 


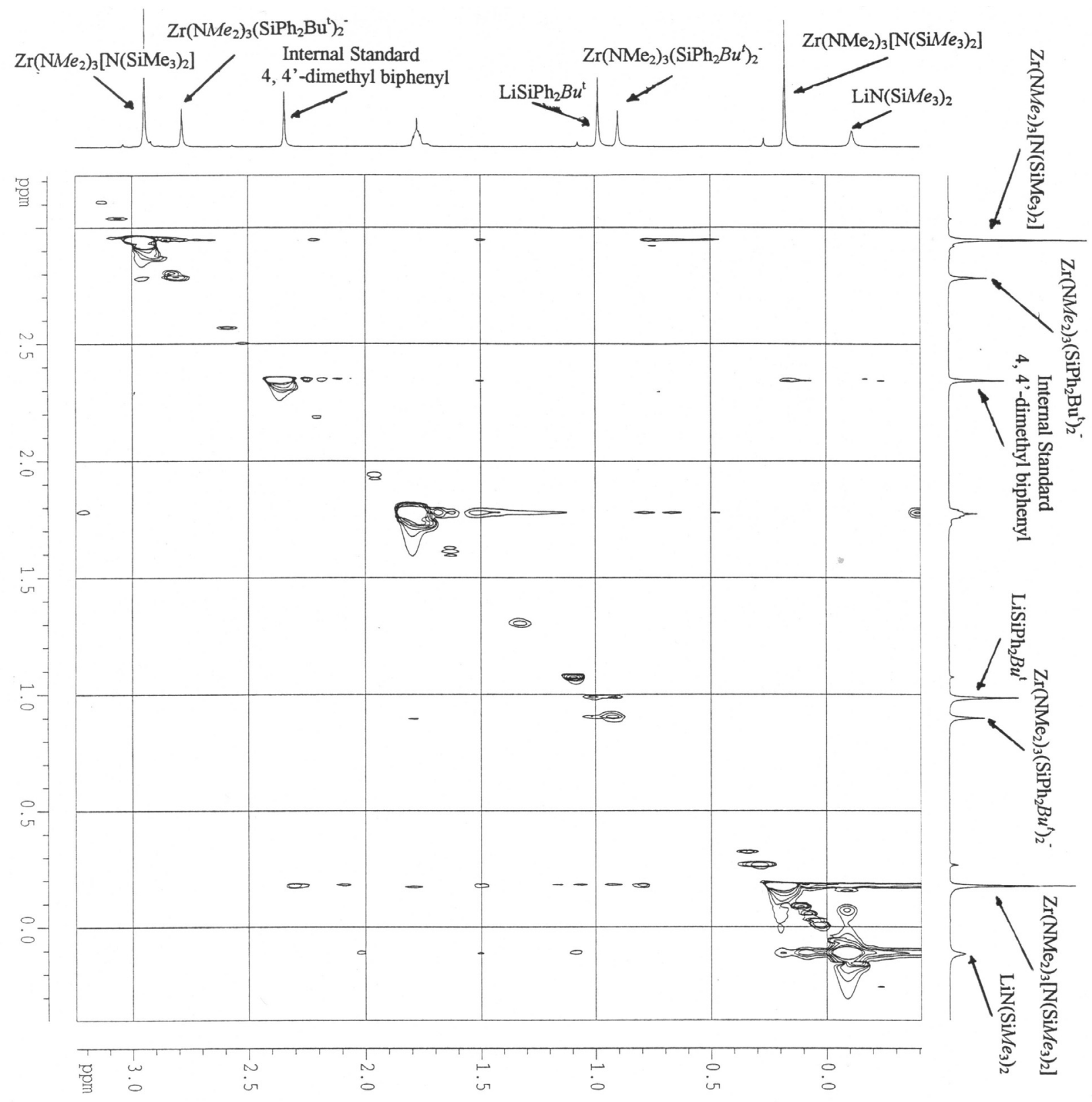

Figure S2. EXSY spectrum (THF- $\left.d_{8}, 400.0 \mathrm{MHz}, 32^{\circ} \mathrm{C}, t_{\text {mix }}=4 \mathrm{~s}\right)$ of a mixture of 2 and 6.

EXSY spectrum (THF- $d_{8}, 32^{\circ} \mathrm{C}$ ) with $t_{\text {mix }}=2 \mathrm{~s}$ did not show crosspeaks. Decomposition of the mixture was observed during the acquisition of the EXSY data at $32{ }^{\circ} \mathrm{C}$, making it difficult to conduct EXSY NMR studies at a higher temperature. 


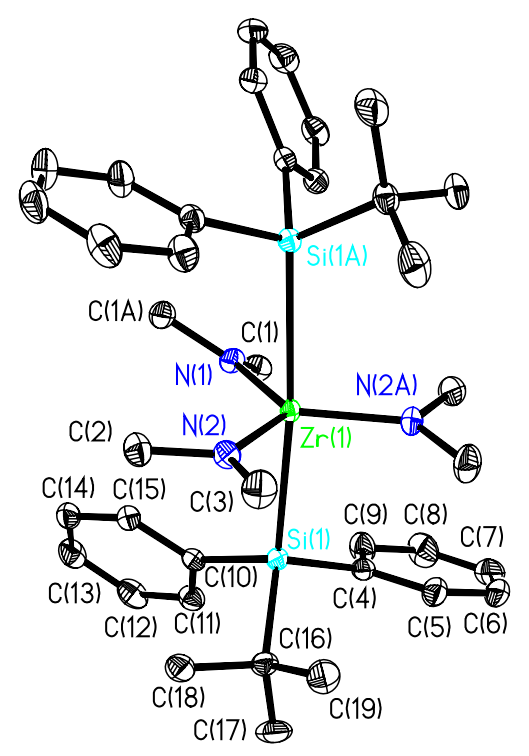

Figure S3. A molecular drawing of the anion 1a in $\mathbf{1}$ shown with $30 \%$ probability ellipsoids. The $\mathrm{H}$ atoms have been omitted for clarity. The structure of this anion (1a) is similar to that reported earlier. $^{6 c}$ 
Identification code

Empirical formula

Formula weight

Temperature

Wavelength

Crystal system

Space group

Unit cell dimensions

Volume

Z

Density (calculated)

Absorption coefficient

$\mathrm{F}(000)$

Crystal size

Theta range for data collection

Index ranges

Reflections collected

Independent reflections

Completeness to theta $=28.28^{\circ}$

Absorption correction

Max. and min. transmission

Refinement method

Data / restraints / parameters

Goodness-of-fit on $\mathrm{F}^{2}$

Final R indices [I $>2$ sigma(I)]

$\mathrm{R}$ indices (all data)

Largest diff. peak and hole
1

C64 H118 Li2 N8 O2 Si2 Zr2

1316.18

173(2) K

$0.71073 \AA$

Monoclinic

$\mathrm{C} 2 / \mathrm{c}$

$\mathrm{a}=25.145(3) \AA$

$\alpha=90^{\circ}$

$\mathrm{b}=17.770(3) \AA$

$\beta=97.789(3)^{\circ}$

$\mathrm{c}=16.346(3) \AA$

$\gamma=90^{\circ}$

4

$1.208 \mathrm{~g} / \mathrm{cm}^{3}$

$0.368 \mathrm{~mm}^{-1}$

2816

$0.40 \times 0.25 \times 0.25 \mathrm{~mm}^{3}$

1.41 to $28.28^{\circ}$

$-32 \unlhd h \unlhd 33,-23 \unlhd k \unlhd 3,-21 \unlhd \unlhd 21$

36482

$8684[\mathrm{R}(\mathrm{int})=0.0543]$

$96.5 \%$

Multi-scan with SADABS

0.9136 and 0.8667

Full-matrix least-squares on $\mathrm{F}^{2}$

8684 / 0 / 383

1.041

$\mathrm{R} 1=0.0396, \mathrm{wR} 2=0.1030$

$\mathrm{R} 1=0.0567, \mathrm{wR} 2=0.1169$

0.875 and -0.464 e. $\AA^{-3}$ 
Table 2. Atomic coordinates $\left(\times 10^{4}\right)$ and equivalent isotropic displacement parameters $\left(\AA^{2}\right.$ $\times 10^{3}$ ) for 1 . $U\left(\right.$ eq) is defined as one third of the trace of the orthogonalized $U^{i j}$ tensor.

\begin{tabular}{|c|c|c|c|c|}
\hline & $\mathrm{x}$ & $\mathrm{y}$ & z & $\mathrm{U}(\mathrm{eq})$ \\
\hline $\operatorname{Zr}(1)$ & 0 & $1532(1)$ & 2500 & $21(1)$ \\
\hline $\operatorname{Zr}(2)$ & 5000 & $1512(1)$ & 2500 & $24(1)$ \\
\hline $\operatorname{Si}(1)$ & $1171(1)$ & $1670(1)$ & $2965(1)$ & $26(1)$ \\
\hline $\mathrm{O}(1)$ & $3564(1)$ & 2192(1) & $1107(1)$ & $49(1)$ \\
\hline $\mathrm{O}(2)$ & $3540(1)$ & $516(1)$ & 663(1) & $49(1)$ \\
\hline $\mathrm{N}(1)$ & 0 & $2702(1)$ & 2500 & $29(1)$ \\
\hline $\mathrm{N}(2)$ & $50(1)$ & $945(1)$ & $1434(1)$ & $32(1)$ \\
\hline $\mathrm{N}(3)$ & 5000 & $2658(2)$ & 2500 & $34(1)$ \\
\hline $\mathrm{N}(4)$ & $4821(1)$ & $1430(1)$ & $1121(1)$ & $35(1)$ \\
\hline $\mathrm{N}(5)$ & $4251(1)$ & 951(1) & $2513(1)$ & $34(1)$ \\
\hline $\operatorname{Li}(1)$ & $4012(2)$ & $1276(2)$ & $1271(3)$ & $37(1)$ \\
\hline$C(1)$ & $232(1)$ & $3162(1)$ & $3187(2)$ & $38(1)$ \\
\hline$C(2)$ & $98(1)$ & $1342(2)$ & $678(2)$ & $42(1)$ \\
\hline$C(3)$ & $5(1)$ & 139(2) & $1298(2)$ & $51(1)$ \\
\hline C(4) & $1353(1)$ & $1680(1)$ & $4145(1)$ & $32(1)$ \\
\hline$C(5)$ & $1322(1)$ & $1013(2)$ & $4602(2)$ & $43(1)$ \\
\hline$C(6)$ & $1402(1)$ & $1004(2)$ & $5461(2)$ & $52(1)$ \\
\hline$C(7)$ & $1517(1)$ & $1655(2)$ & $5901(2)$ & $56(1)$ \\
\hline $\mathrm{C}(8)$ & $1545(1)$ & $2321(2)$ & $5473(2)$ & $59(1)$ \\
\hline C(9) & $1458(1)$ & $2332(2)$ & $4616(2)$ & $46(1)$ \\
\hline$C(10)$ & $1479(1)$ & $2582(1)$ & $2599(1)$ & $29(1)$ \\
\hline$C(11)$ & $1947(1)$ & $2938(2)$ & $2975(2)$ & $38(1)$ \\
\hline$C(12)$ & $2135(1)$ & $3607(2)$ & $2666(2)$ & $48(1)$ \\
\hline$C(13)$ & $1867(1)$ & $3930(2)$ & $1961(2)$ & $47(1)$ \\
\hline$C(14)$ & 1413(1) & $3587(1)$ & $1564(2)$ & $40(1)$ \\
\hline$C(15)$ & $1223(1)$ & 2931(1) & 1883(1) & $32(1)$ \\
\hline$C(16)$ & $1640(1)$ & $910(1)$ & $2561(2)$ & $35(1)$ \\
\hline$C(17)$ & $2223(1)$ & $967(2)$ & $2975(2)$ & $55(1)$ \\
\hline $\mathrm{C}(18)$ & $1629(1)$ & $1039(2)$ & $1634(2)$ & $43(1)$ \\
\hline$C(19)$ & $1429(1)$ & $113(2)$ & $2672(2)$ & $58(1)$ \\
\hline$C(20)$ & $4554(1)$ & $3120(2)$ & $2656(3)$ & $68(1)$ \\
\hline$C(21)$ & $4906(1)$ & $743(2)$ & $672(2)$ & $54(1)$ \\
\hline$C(22)$ & $4924(1)$ & $2059(2)$ & 591(2) & $53(1)$ \\
\hline$C(23)$ & $4248(1)$ & $127(2)$ & $2474(2)$ & $57(1)$ \\
\hline$C(24)$ & $3880(1)$ & $1204(2)$ & $3069(2)$ & $50(1)$ \\
\hline$C(25)$ & $2982(1)$ & 593(2) & $677(3)$ & $86(1)$ \\
\hline$C(26)$ & $2700(1)$ & $-5(2)$ & $166(2)$ & $63(1)$ \\
\hline$C(27)$ & $3124(1)$ & $-569(2)$ & $77(3)$ & $70(1)$ \\
\hline $\mathrm{C}(28)$ & $3637(1)$ & $-204(2)$ & $312(3)$ & $83(1)$ \\
\hline$C(29)$ & $3568(2)$ & $2640(2)$ & $371(3)$ & $82(1)$ \\
\hline
\end{tabular}




\begin{tabular}{lrrrr}
$\mathrm{C}(30)$ & $3368(3)$ & $3369(3)$ & $514(3)$ & $113(2)$ \\
$\mathrm{C}(31)$ & $3063(2)$ & $3290(3)$ & $1206(3)$ & $108(2)$ \\
$\mathrm{C}(32)$ & $3160(1)$ & $2513(2)$ & $1548(2)$ & $56(1)$ \\
\hline
\end{tabular}


Table 3. Bond lengths $(\AA)$ and angles $\left({ }^{\circ}\right)$ for 1

\begin{tabular}{|c|c|c|c|}
\hline $\mathrm{Zr}(1)-\mathrm{N}(2)$ & $2.0488(19)$ & $\mathrm{N}(4)-\mathrm{C}(21)$ & $1.454(3)$ \\
\hline $\mathrm{Zr}(1)-\mathrm{N}(2) \# 1$ & $2.0488(19)$ & $\mathrm{N}(4)-\mathrm{C}(22)$ & $1.459(3)$ \\
\hline $\mathrm{Zr}(1)-\mathrm{N}(1)$ & $2.080(2)$ & $\mathrm{N}(4)-\mathrm{Li}(1)$ & $2.099(4)$ \\
\hline $\operatorname{Zr}(1)-\operatorname{Si}(1) \# 1$ & $2.9507(7)$ & $\mathrm{N}(5)-\mathrm{C}(24)$ & $1.459(3)$ \\
\hline $\operatorname{Zr}(1)-\operatorname{Si}(1)$ & $2.9507(7)$ & $\mathrm{N}(5)-\mathrm{C}(23)$ & $1.465(3)$ \\
\hline $\mathrm{Zr}(2)-\mathrm{N}(3)$ & $2.036(3)$ & N(5)-Li(1) & $2.117(5)$ \\
\hline $\mathrm{Zr}(2)-\mathrm{N}(5)$ & $2.1337(19)$ & $C(4)-C(9)$ & $1.397(4)$ \\
\hline $\mathrm{Zr}(2)-\mathrm{N}(5) \# 2$ & $2.1337(19)$ & $C(4)-C(5)$ & $1.408(3)$ \\
\hline $\mathrm{Zr}(2)-\mathrm{N}(4)$ & $2.243(2)$ & $C(5)-C(6)$ & $1.392(4)$ \\
\hline $\mathrm{Zr}(2)-\mathrm{N}(4) \# 2$ & $2.243(2)$ & $C(6)-C(7)$ & $1.373(5)$ \\
\hline $\operatorname{Zr}(2)-\operatorname{Li}(1) \# 2$ & $3.005(4)$ & $\mathrm{C}(7)-\mathrm{C}(8)$ & $1.380(5)$ \\
\hline $\operatorname{Zr}(2)-\operatorname{Li}(1)$ & $3.005(4)$ & $\mathrm{C}(8)-\mathrm{C}(9)$ & $1.389(4)$ \\
\hline $\operatorname{Si}(1)-C(4)$ & $1.920(2)$ & $C(10)-C(15)$ & $1.400(3)$ \\
\hline $\operatorname{Si}(1)-C(10)$ & $1.926(2)$ & $\mathrm{C}(10)-\mathrm{C}(11)$ & $1.403(3)$ \\
\hline $\mathrm{Si}(1)-\mathrm{C}(16)$ & $1.965(2)$ & $\mathrm{C}(11)-\mathrm{C}(12)$ & $1.399(4)$ \\
\hline $\mathrm{O}(1)-\mathrm{C}(32)$ & $1.440(3)$ & $C(12)-C(13)$ & $1.379(4)$ \\
\hline $\mathrm{O}(1)-\mathrm{C}(29)$ & $1.442(4)$ & $C(13)-C(14)$ & $1.375(4)$ \\
\hline $\mathrm{O}(1)-\mathrm{Li}(1)$ & $1.979(5)$ & $C(14)-C(15)$ & $1.388(3)$ \\
\hline $\mathrm{O}(2)-\mathrm{C}(25)$ & $1.413(3)$ & $\mathrm{C}(16)-\mathrm{C}(18)$ & $1.529(4)$ \\
\hline $\mathrm{O}(2)-\mathrm{C}(28)$ & $1.437(3)$ & $C(16)-C(17)$ & $1.531(4)$ \\
\hline $\mathrm{O}(2)-\mathrm{Li}(1)$ & $1.975(5)$ & $C(16)-C(19)$ & $1.531(4)$ \\
\hline $\mathrm{N}(1)-\mathrm{C}(1) \# 1$ & $1.447(3)$ & $C(25)-C(26)$ & $1.471(4)$ \\
\hline $\mathrm{N}(1)-\mathrm{C}(1)$ & $1.447(3)$ & $C(26)-C(27)$ & $1.485(5)$ \\
\hline $\mathrm{N}(2)-\mathrm{C}(2)$ & $1.442(3)$ & $\mathrm{C}(27)-\mathrm{C}(28)$ & $1.450(5)$ \\
\hline $\mathrm{N}(2)-\mathrm{C}(3)$ & $1.452(3)$ & $C(29)-C(30)$ & $1.420(5)$ \\
\hline $\mathrm{N}(3)-\mathrm{C}(20) \# 2$ & $1.440(3)$ & $\mathrm{C}(30)-\mathrm{C}(31)$ & $1.456(6)$ \\
\hline $\mathrm{N}(3)-\mathrm{C}(20)$ & $1.440(3)$ & $\mathrm{C}(31)-\mathrm{C}(32)$ & $1.497(5)$ \\
\hline $\mathrm{N}(2)-\operatorname{Zr}(1)-\mathrm{N}(2) \# 1$ & $118.86(11)$ & $\mathrm{N}(3)-\mathrm{Zr}(2)-\mathrm{N}(4) \# 2$ & $93.73(5)$ \\
\hline $\mathrm{N}(2)-\mathrm{Zr}(1)-\mathrm{N}(1)$ & $120.57(6)$ & $\mathrm{N}(5)-\mathrm{Zr}(2)-\mathrm{N}(4) \# 2$ & $90.97(8)$ \\
\hline $\mathrm{N}(2) \# 1-\operatorname{Zr}(1)-\mathrm{N}(1)$ & $120.57(6)$ & $\mathrm{N}(5) \# 2-\mathrm{Zr}(2)-\mathrm{N}(4) \# 2$ & $85.54(8)$ \\
\hline $\mathrm{N}(2)-\operatorname{Zr}(1)-\mathrm{Si}(1) \# 1$ & $89.83(6)$ & $\mathrm{N}(4)-\mathrm{Zr}(2)-\mathrm{N}(4) \# 2$ & $172.54(11)$ \\
\hline $\mathrm{N}(2) \# 1-\mathrm{Zr}(1)-\mathrm{Si}(1) \# 1$ & $95.01(6)$ & $N(3)-\operatorname{Zr}(2)-\operatorname{Li}(1) \# 2$ & $98.03(8)$ \\
\hline $\mathrm{N}(1)-\operatorname{Zr}(1)-\operatorname{Si}(1) \# 1$ & $85.235(13)$ & $\mathrm{N}(5)-\operatorname{Zr}(2)-\mathrm{Li}(1) \# 2$ & $125.38(10)$ \\
\hline $\mathrm{N}(2)-\mathrm{Zr}(1)-\mathrm{Si}(1)$ & $95.01(6)$ & $\mathrm{N}(5) \# 2-\operatorname{Zr}(2)-\mathrm{Li}(1) \# 2$ & $44.80(10)$ \\
\hline $\mathrm{N}(2) \# 1-\mathrm{Zr}(1)-\mathrm{Si}(1)$ & $89.83(6)$ & $\mathrm{N}(4)-\operatorname{Zr}(2)-\mathrm{Li}(1) \# 2$ & $134.27(10)$ \\
\hline $\mathrm{N}(1)-\operatorname{Zr}(1)-\operatorname{Si}(1)$ & $85.235(13)$ & $\mathrm{N}(4) \# 2-\operatorname{Zr}(2)-\operatorname{Li}(1) \# 2$ & $44.26(9)$ \\
\hline $\operatorname{Si}(1) \# 1-\operatorname{Zr}(1)-\operatorname{Si}(1)$ & $170.47(3)$ & $\mathrm{N}(3)-\operatorname{Zr}(2)-\operatorname{Li}(1)$ & $98.03(8)$ \\
\hline $\mathrm{N}(3)-\operatorname{Zr}(2)-\mathrm{N}(5)$ & $117.85(5)$ & $N(5)-\operatorname{Zr}(2)-\operatorname{Li}(1)$ & $44.80(10)$ \\
\hline $\mathrm{N}(3)-\mathrm{Zr}(2)-\mathrm{N}(5) \# 2$ & $117.85(5)$ & $\mathrm{N}(5) \# 2-\mathrm{Zr}(2)-\mathrm{Li}(1)$ & $125.38(10)$ \\
\hline $\mathrm{N}(5)-\mathrm{Zr}(2)-\mathrm{N}(5) \# 2$ & $124.30(11)$ & $N(4)-\operatorname{Zr}(2)-\operatorname{Li}(1)$ & $44.26(9)$ \\
\hline $\mathrm{N}(3)-\mathrm{Zr}(2)-\mathrm{N}(4)$ & $93.73(5)$ & $\mathrm{N}(4) \# 2-\mathrm{Zr}(2)-\mathrm{Li}(1)$ & $134.27(10)$ \\
\hline $\mathrm{N}(5)-\mathrm{Zr}(2)-\mathrm{N}(4)$ & $85.54(8)$ & $\mathrm{Li}(1) \# 2-\operatorname{Zr}(2)-\mathrm{Li}(1)$ & $163.93(16)$ \\
\hline $\mathrm{N}(5) \# 2-\mathrm{Zr}(2)-\mathrm{N}(4)$ & $90.97(8)$ & $C(4)-\operatorname{Si}(1)-C(10)$ & $104.70(10)$ \\
\hline
\end{tabular}




\begin{tabular}{|c|c|c|c|}
\hline C(4)-Si(1)-C(16) & $105.79(10)$ & $\mathrm{O}(1)-\mathrm{Li}(1)-\mathrm{N}(5)$ & $115.9(2)$ \\
\hline$C(10)-\operatorname{Si}(1)-C(16)$ & $100.95(10)$ & N(4)-Li(1)-N(5) & $89.68(18)$ \\
\hline $\mathrm{C}(4)-\mathrm{Si}(1)-\operatorname{Zr}(1)$ & $110.65(7)$ & $\mathrm{O}(2)-\mathrm{Li}(1)-\mathrm{Zr}(2)$ & $144.9(2)$ \\
\hline C(10)-Si(1)-Zr(1) & $114.71(7)$ & $\mathrm{O}(1)-\mathrm{Li}(1)-\mathrm{Zr}(2)$ & $112.35(18)$ \\
\hline C(16)-Si(1)-Zr(1) & $118.66(8)$ & $\mathrm{N}(4)-\operatorname{Li}(1)-\operatorname{Zr}(2)$ & $48.23(10)$ \\
\hline $\mathrm{C}(32)-\mathrm{O}(1)-\mathrm{C}(29)$ & $106.5(2)$ & $\mathrm{N}(5)-\mathrm{Li}(1)-\mathrm{Zr}(2)$ & $45.24(10)$ \\
\hline $\mathrm{C}(32)-\mathrm{O}(1)-\mathrm{Li}(1)$ & $133.2(2)$ & $C(9)-C(4)-C(5)$ & $115.1(2)$ \\
\hline C(29)-O(1)-Li(1) & $119.9(2)$ & $\mathrm{C}(9)-\mathrm{C}(4)-\mathrm{Si}(1)$ & $124.25(19)$ \\
\hline $\mathrm{C}(25)-\mathrm{O}(2)-\mathrm{C}(28)$ & $108.4(2)$ & $\mathrm{C}(5)-\mathrm{C}(4)-\mathrm{Si}(1)$ & $120.18(19)$ \\
\hline $\mathrm{C}(25)-\mathrm{O}(2)-\mathrm{Li}(1)$ & $117.2(2)$ & $C(6)-C(5)-C(4)$ & $122.4(3)$ \\
\hline $\mathrm{C}(28)-\mathrm{O}(2)-\mathrm{Li}(1)$ & $133.3(2)$ & $C(7)-C(6)-C(5)$ & $120.6(3)$ \\
\hline $\mathrm{C}(1) \# 1-\mathrm{N}(1)-\mathrm{C}(1)$ & $111.2(3)$ & $C(6)-C(7)-C(8)$ & $118.6(3)$ \\
\hline $\mathrm{C}(1) \# 1-\mathrm{N}(1)-\mathrm{Zr}(1)$ & $124.40(13)$ & $\mathrm{C}(7)-\mathrm{C}(8)-\mathrm{C}(9)$ & $120.8(3)$ \\
\hline $\mathrm{C}(1)-\mathrm{N}(1)-\mathrm{Zr}(1)$ & $124.40(13)$ & $C(8)-C(9)-C(4)$ & $122.5(3)$ \\
\hline $\mathrm{C}(2)-\mathrm{N}(2)-\mathrm{C}(3)$ & $111.4(2)$ & $C(15)-C(10)-C(11)$ & $115.4(2)$ \\
\hline $\mathrm{C}(2)-\mathrm{N}(2)-\mathrm{Zr}(1)$ & $120.17(16)$ & $C(15)-C(10)-\operatorname{Si}(1)$ & $118.34(17)$ \\
\hline $\mathrm{C}(3)-\mathrm{N}(2)-\mathrm{Zr}(1)$ & $128.32(17)$ & C(11)-C(10)-Si(1) & $126.25(18)$ \\
\hline $\mathrm{C}(20) \# 2-\mathrm{N}(3)-\mathrm{C}(20)$ & $110.4(3)$ & $C(12)-C(11)-C(10)$ & $122.0(3)$ \\
\hline $\mathrm{C}(20) \# 2-\mathrm{N}(3)-\mathrm{Zr}(2)$ & $124.79(16)$ & $C(13)-C(12)-C(11)$ & $120.2(3)$ \\
\hline $\mathrm{C}(20)-\mathrm{N}(3)-\mathrm{Zr}(2)$ & $124.79(16)$ & $C(14)-C(13)-C(12)$ & $119.4(2)$ \\
\hline $\mathrm{C}(21)-\mathrm{N}(4)-\mathrm{C}(22)$ & $107.0(2)$ & $C(13)-C(14)-C(15)$ & $120.1(3)$ \\
\hline $\mathrm{C}(21)-\mathrm{N}(4)-\mathrm{Li}(1)$ & $99.3(2)$ & $C(14)-C(15)-C(10)$ & $122.9(2)$ \\
\hline $\mathrm{C}(22)-\mathrm{N}(4)-\mathrm{Li}(1)$ & $115.0(2)$ & $C(18)-C(16)-C(17)$ & $108.4(2)$ \\
\hline $\mathrm{C}(21)-\mathrm{N}(4)-\operatorname{Zr}(2)$ & $122.56(18)$ & $C(18)-C(16)-C(19)$ & $107.2(2)$ \\
\hline $\mathrm{C}(22)-\mathrm{N}(4)-\operatorname{Zr}(2)$ & $121.29(17)$ & $C(17)-C(16)-C(19)$ & $109.6(2)$ \\
\hline $\mathrm{Li}(1)-\mathrm{N}(4)-\mathrm{Zr}(2)$ & $87.51(14)$ & $C(18)-C(16)-\operatorname{Si}(1)$ & $107.36(16)$ \\
\hline $\mathrm{C}(24)-\mathrm{N}(5)-\mathrm{C}(23)$ & $109.6(2)$ & $C(17)-C(16)-\operatorname{Si}(1)$ & $112.81(18)$ \\
\hline $\mathrm{C}(24)-\mathrm{N}(5)-\mathrm{Li}(1)$ & $113.0(2)$ & $C(19)-C(16)-\operatorname{Si}(1)$ & $111.21(18)$ \\
\hline $\mathrm{C}(23)-\mathrm{N}(5)-\mathrm{Li}(1)$ & $103.4(2)$ & $\mathrm{O}(2)-\mathrm{C}(25)-\mathrm{C}(26)$ & $108.9(3)$ \\
\hline $\mathrm{C}(24)-\mathrm{N}(5)-\operatorname{Zr}(2)$ & $120.11(18)$ & $C(25)-C(26)-C(27)$ & $104.2(3)$ \\
\hline $\mathrm{C}(23)-\mathrm{N}(5)-\mathrm{Zr}(2)$ & $117.76(17)$ & $C(28)-C(27)-C(26)$ & $107.3(3)$ \\
\hline $\mathrm{Li}(1)-\mathrm{N}(5)-\mathrm{Zr}(2)$ & $89.96(13)$ & $\mathrm{O}(2)-\mathrm{C}(28)-\mathrm{C}(27)$ & $108.0(3)$ \\
\hline $\mathrm{O}(2)-\mathrm{Li}(1)-\mathrm{O}(1)$ & $101.6(2)$ & $\mathrm{C}(30)-\mathrm{C}(29)-\mathrm{O}(1)$ & $108.9(3)$ \\
\hline $\mathrm{O}(2)-\mathrm{Li}(1)-\mathrm{N}(4)$ & $123.8(2)$ & $\mathrm{C}(29)-\mathrm{C}(30)-\mathrm{C}(31)$ & $105.9(4)$ \\
\hline $\mathrm{O}(1)-\mathrm{Li}(1)-\mathrm{N}(4)$ & $114.8(2)$ & $\mathrm{C}(30)-\mathrm{C}(31)-\mathrm{C}(32)$ & $107.7(3)$ \\
\hline $\mathrm{O}(2)-\mathrm{Li}(1)-\mathrm{N}(5)$ & $111.7(2)$ & $\mathrm{O}(1)-\mathrm{C}(32)-\mathrm{C}(31)$ & $105.5(3)$ \\
\hline
\end{tabular}

Symmetry transformations used to generate equivalent atoms:

$\# 1-\mathrm{x}, \mathrm{y},-\mathrm{z}+1 / 2 \quad \# 2-\mathrm{x}+1, \mathrm{y},-\mathrm{z}+1 / 2$ 
Table 4. Anisotropic displacement parameters $\left(\AA^{2} \times 10^{3}\right)$ for 1 . The anisotropic displacement factor exponent takes the form: $-2 \pi^{2}\left(h^{2} a^{* 2} U^{11}+\ldots+2 h k a * b * U^{12}\right)$

\begin{tabular}{|c|c|c|c|c|c|c|}
\hline & $\mathrm{U}^{11}$ & $\mathrm{U}^{22}$ & $\mathrm{U}^{33}$ & $\mathrm{U}^{23}$ & $\mathrm{U}^{13}$ & $\mathrm{U}^{12}$ \\
\hline $\operatorname{Zr}(1)$ & $26(1)$ & $17(1)$ & $21(1)$ & 0 & $5(1)$ & 0 \\
\hline $\operatorname{Zr}(2)$ & $27(1)$ & $19(1)$ & $27(1)$ & 0 & $8(1)$ & 0 \\
\hline $\operatorname{Si}(1)$ & $26(1)$ & $26(1)$ & $25(1)$ & $1(1)$ & $4(1)$ & $3(1)$ \\
\hline $\mathrm{O}(1)$ & $56(1)$ & $40(1)$ & $55(1)$ & $-2(1)$ & $16(1)$ & $15(1)$ \\
\hline $\mathrm{O}(2)$ & $35(1)$ & $47(1)$ & $64(1)$ & $-23(1)$ & $1(1)$ & $1(1)$ \\
\hline $\mathrm{N}(1)$ & $32(1)$ & $17(1)$ & $39(2)$ & 0 & $6(1)$ & 0 \\
\hline $\mathrm{N}(2)$ & $37(1)$ & $32(1)$ & $28(1)$ & $-6(1)$ & $5(1)$ & $2(1)$ \\
\hline $\mathrm{N}(3)$ & $41(2)$ & $22(1)$ & $41(2)$ & 0 & $8(1)$ & 0 \\
\hline $\mathrm{N}(4)$ & $36(1)$ & $40(1)$ & $30(1)$ & $-2(1)$ & $10(1)$ & $1(1)$ \\
\hline $\mathrm{N}(5)$ & $34(1)$ & $31(1)$ & $39(1)$ & $0(1)$ & $11(1)$ & $-6(1)$ \\
\hline $\operatorname{Li}(1)$ & $32(2)$ & $34(2)$ & $44(2)$ & $-6(2)$ & $6(2)$ & $0(2)$ \\
\hline $\mathrm{C}(1)$ & $38(1)$ & $29(1)$ & $47(2)$ & $-7(1)$ & $8(1)$ & $0(1)$ \\
\hline$C(2)$ & $45(2)$ & $48(2)$ & $34(1)$ & $0(1)$ & $8(1)$ & $-5(1)$ \\
\hline$C(3)$ & $72(2)$ & $32(1)$ & $50(2)$ & $-8(1)$ & $12(1)$ & $3(1)$ \\
\hline$C(4)$ & $27(1)$ & $40(1)$ & $27(1)$ & $2(1)$ & $4(1)$ & $6(1)$ \\
\hline$C(5)$ & $50(2)$ & $46(2)$ & $34(1)$ & $6(1)$ & $6(1)$ & $7(1)$ \\
\hline$C(6)$ & $56(2)$ & $67(2)$ & $35(2)$ & $18(1)$ & $11(1)$ & $22(1)$ \\
\hline$C(7)$ & $51(2)$ & $92(2)$ & $26(1)$ & $1(2)$ & $6(1)$ & $23(2)$ \\
\hline $\mathrm{C}(8)$ & $72(2)$ & $70(2)$ & $36(2)$ & $-15(1)$ & $11(1)$ & $-1(2)$ \\
\hline $\mathrm{C}(9)$ & $59(2)$ & $47(2)$ & $34(1)$ & $-4(1)$ & $14(1)$ & $1(1)$ \\
\hline$C(10)$ & $28(1)$ & $31(1)$ & $28(1)$ & $-3(1)$ & $9(1)$ & $2(1)$ \\
\hline $\mathrm{C}(11)$ & $30(1)$ & $45(1)$ & $37(1)$ & $-3(1)$ & $5(1)$ & $-2(1)$ \\
\hline$C(12)$ & $38(1)$ & $50(2)$ & $57(2)$ & $-11(1)$ & $14(1)$ & $-16(1)$ \\
\hline$C(13)$ & $51(2)$ & $34(1)$ & $59(2)$ & $2(1)$ & $23(1)$ & $-6(1)$ \\
\hline$C(14)$ & $44(1)$ & $36(1)$ & $42(1)$ & $8(1)$ & $15(1)$ & $5(1)$ \\
\hline$C(15)$ & $32(1)$ & $33(1)$ & $32(1)$ & $2(1)$ & $6(1)$ & $0(1)$ \\
\hline$C(16)$ & $36(1)$ & $35(1)$ & $34(1)$ & $-1(1)$ & $5(1)$ & $10(1)$ \\
\hline$C(17)$ & $39(2)$ & $73(2)$ & $52(2)$ & $-5(2)$ & $0(1)$ & $27(1)$ \\
\hline $\mathrm{C}(18)$ & $49(2)$ & $45(2)$ & $35(1)$ & $-6(1)$ & $10(1)$ & $12(1)$ \\
\hline C(19) & $82(2)$ & $33(1)$ & $64(2)$ & $2(1)$ & $28(2)$ & $15(1)$ \\
\hline $\mathrm{C}(20)$ & $54(2)$ & $38(2)$ & $114(3)$ & $-10(2)$ & $25(2)$ & $5(1)$ \\
\hline $\mathrm{C}(21)$ & $52(2)$ & $66(2)$ & $44(2)$ & $-21(2)$ & $7(1)$ & $9(2)$ \\
\hline$C(22)$ & $57(2)$ & $70(2)$ & $33(1)$ & $12(1)$ & $10(1)$ & $-11(2)$ \\
\hline$C(23)$ & $58(2)$ & $34(2)$ & $76(2)$ & $6(1)$ & $3(2)$ & $-13(1)$ \\
\hline$C(24)$ & $37(1)$ & $69(2)$ & $48(2)$ & $12(1)$ & 17(1) & $4(1)$ \\
\hline$C(25)$ & $35(2)$ & $86(3)$ & $136(4)$ & $-63(3)$ & $9(2)$ & $-4(2)$ \\
\hline$C(26)$ & $45(2)$ & $63(2)$ & $79(2)$ & $-23(2)$ & $4(2)$ & $-8(1)$ \\
\hline$C(27)$ & $58(2)$ & $43(2)$ & $106(3)$ & $-17(2)$ & $-4(2)$ & $-3(2)$ \\
\hline $\mathrm{C}(28)$ & $56(2)$ & $68(2)$ & $120(3)$ & $-58(2)$ & $-11(2)$ & $12(2)$ \\
\hline$C(29)$ & $108(3)$ & $76(3)$ & $72(2)$ & $17(2)$ & $47(2)$ & $37(2)$ \\
\hline
\end{tabular}




\begin{tabular}{lcccccc}
$\mathrm{C}(30)$ & $188(6)$ & $87(3)$ & $76(3)$ & $32(2)$ & $61(3)$ & $70(3)$ \\
$\mathrm{C}(31)$ & $148(5)$ & $89(3)$ & $102(3)$ & $29(3)$ & $72(3)$ & $66(3)$ \\
$\mathrm{C}(32)$ & $59(2)$ & $55(2)$ & $58(2)$ & $-3(1)$ & $23(2)$ & $13(2)$ \\
\hline
\end{tabular}


Table 5. Hydrogen coordinates $\left(\times 10^{4}\right)$ and isotropic displacement parameters $\left(\AA^{2} \times 10^{3}\right)$ for 1

\begin{tabular}{|c|c|c|c|c|}
\hline & $\mathrm{x}$ & $\mathrm{y}$ & $\mathrm{z}$ & $\mathrm{U}(\mathrm{eq})$ \\
\hline $\mathrm{H}(1 \mathrm{~A})$ & 524 & 3467 & 3019 & 57 \\
\hline $\mathrm{H}(1 \mathrm{~B})$ & 375 & 2837 & 3650 & 57 \\
\hline $\mathrm{H}(1 \mathrm{C})$ & -44 & 3494 & 3358 & 57 \\
\hline $\mathrm{H}(2 \mathrm{~A})$ & 415 & 1161 & 449 & 63 \\
\hline $\mathrm{H}(2 \mathrm{~B})$ & 134 & 1882 & 791 & 63 \\
\hline $\mathrm{H}(2 \mathrm{C})$ & -224 & 1252 & 279 & 63 \\
\hline $\mathrm{H}(3 \mathrm{~A})$ & -294 & 35 & 863 & 76 \\
\hline $\mathrm{H}(3 \mathrm{~B})$ & -59 & -110 & 1810 & 76 \\
\hline $\mathrm{H}(3 \mathrm{C})$ & 339 & -52 & 1128 & 76 \\
\hline $\mathrm{H}(5)$ & 1244 & 554 & 4314 & 52 \\
\hline $\mathrm{H}(6)$ & 1377 & 542 & 5746 & 63 \\
\hline $\mathrm{H}(7)$ & 1576 & 1648 & 6487 & 67 \\
\hline $\mathrm{H}(8)$ & 1624 & 2776 & 5768 & 71 \\
\hline $\mathrm{H}(9)$ & 1471 & 2800 & 4339 & 55 \\
\hline $\mathrm{H}(11)$ & 2142 & 2718 & 3454 & 45 \\
\hline $\mathrm{H}(12)$ & 2450 & 3839 & 2942 & 57 \\
\hline $\mathrm{H}(13)$ & 1993 & 4385 & 1752 & 56 \\
\hline $\mathrm{H}(14)$ & 1230 & 3799 & 1072 & 48 \\
\hline $\mathrm{H}(15)$ & 906 & 2708 & 1603 & 39 \\
\hline $\mathrm{H}(17 \mathrm{~A})$ & 2441 & 590 & 2737 & 83 \\
\hline $\mathrm{H}(17 \mathrm{~B})$ & 2238 & 877 & 3569 & 83 \\
\hline $\mathrm{H}(17 \mathrm{C})$ & 2362 & 1471 & 2883 & 83 \\
\hline $\mathrm{H}(18 \mathrm{~A})$ & 1844 & 651 & 1407 & 64 \\
\hline $\mathrm{H}(18 \mathrm{~B})$ & 1778 & 1537 & 1542 & 64 \\
\hline $\mathrm{H}(18 \mathrm{C})$ & 1258 & 1013 & 1361 & 64 \\
\hline $\mathrm{H}(19 \mathrm{~A})$ & 1054 & 81 & 2416 & 87 \\
\hline $\mathrm{H}(19 \mathrm{~B})$ & 1452 & -1 & 3262 & 87 \\
\hline $\mathrm{H}(19 \mathrm{C})$ & 1645 & -249 & 2409 & 87 \\
\hline $\mathrm{H}(20 \mathrm{~A})$ & 4663 & 3439 & 3139 & 101 \\
\hline $\mathrm{H}(20 \mathrm{~B})$ & 4255 & 2798 & 2762 & 101 \\
\hline $\mathrm{H}(20 \mathrm{C})$ & 4442 & 3438 & 2174 & 101 \\
\hline $\mathrm{H}(21 \mathrm{~A})$ & 4655 & 728 & 158 & 81 \\
\hline $\mathrm{H}(21 \mathrm{~B})$ & 4846 & 306 & 1013 & 81 \\
\hline $\mathrm{H}(21 \mathrm{C})$ & 5275 & 733 & 542 & 81 \\
\hline $\mathrm{H}(22 \mathrm{~A})$ & 5303 & 2057 & 510 & 79 \\
\hline $\mathrm{H}(22 \mathrm{~B})$ & 4840 & 2533 & 851 & 79 \\
\hline $\mathrm{H}(22 \mathrm{C})$ & 4699 & 2009 & 55 & 79 \\
\hline $\mathrm{H}(23 \mathrm{~A})$ & 4328 & -79 & 3034 & 85 \\
\hline $\mathrm{H}(23 \mathrm{~B})$ & 4520 & -44 & 2141 & 85 \\
\hline $\mathrm{H}(23 \mathrm{C})$ & 3893 & -47 & 2223 & 85 \\
\hline $\mathrm{H}(24 \mathrm{~A})$ & 3513 & 1061 & 2843 & 75 \\
\hline
\end{tabular}




\begin{tabular}{lrrrr}
$\mathrm{H}(24 \mathrm{~B})$ & 3903 & 1752 & 3127 & 75 \\
$\mathrm{H}(24 \mathrm{C})$ & 3976 & 969 & 3612 & 75 \\
$\mathrm{H}(25 \mathrm{~A})$ & 2860 & 1092 & 458 & 103 \\
$\mathrm{H}(25 \mathrm{~B})$ & 2901 & 552 & 1251 & 103 \\
$\mathrm{H}(26 \mathrm{~A})$ & 2543 & 194 & -379 & 75 \\
$\mathrm{H}(26 \mathrm{~B})$ & 2410 & -227 & 442 & 75 \\
$\mathrm{H}(27 \mathrm{~A})$ & 3086 & -1008 & 439 & 84 \\
$\mathrm{H}(27 \mathrm{~B})$ & 3093 & -747 & -501 & 84 \\
$\mathrm{H}(28 \mathrm{~A})$ & 3866 & -516 & 721 & 100 \\
$\mathrm{H}(28 \mathrm{~B})$ & 3825 & -139 & -178 & 100 \\
$\mathrm{H}(29 \mathrm{~A})$ & 3938 & 2677 & 233 & 99 \\
$\mathrm{H}(29 \mathrm{~B})$ & 3340 & 2400 & -99 & 99 \\
$\mathrm{H}(30 \mathrm{~A})$ & 3134 & 3553 & 18 & 136 \\
$\mathrm{H}(30 \mathrm{~B})$ & 3667 & 3729 & 650 & 136 \\
$\mathrm{H}(31 \mathrm{~A})$ & 2676 & 3366 & 1019 & 130 \\
$\mathrm{H}(31 \mathrm{~B})$ & 3183 & 3669 & 1636 & 130 \\
$\mathrm{H}(32 \mathrm{~A})$ & 3289 & 2533 & 2148 & 67 \\
$\mathrm{H}(32 \mathrm{~B})$ & 2826 & 2212 & 1459 & 67 \\
& & & & \\
\hline
\end{tabular}

